\title{
Species Distribution and Antifungal Drug Susceptibility of Candida in Clinical Isolates from a Tertiary Care Centre at Bareilly
}

\author{
Seema Gupta ${ }^{1}$, Rahul Kumar Goyal ${ }^{2}$ \\ ${ }^{1,2}$ Department Of Microbiology, Shri Ram Murti Smarak Institute Of Medical Sciences, (SRMS IMS) \\ Bareilly U.P. India
}

\begin{abstract}
Background: The incidence of fungal infections has increased significantly, causing increased morbidity and mortality. The important factor for increased incidence of fungal infections is the overuse of broad spectrum antibiotics for the treatment of multidrug resistant bacteria.. Candida is the major fungal pathogen that causes both mucosal and deep tissue infections.

Aim:The aim of our study is to identify the Candida species among clinical isolates and their sensitivity pattern for the common antifungal drugs.

Material and methods: Eighty nine different clinical isolates of Candida species were collected from patients admitted in the I.C.U. from 15 jan 2016 to 15 july 2016. The identification of fungal species as well as antifungal sensitivity testing was performed with Vitek 2 compact ( Biomerieux France) using Vitek 2 cards for identification of yeast and yeast like organisms. (ID YST Card). Antifungal susceptibility test was performed with Vitek 2 Fungal susceptibility card (AST YSO7) kit respectively.

Result: In this study we found $31.46 \%$ isolates were Candida albicans and $68.53 \%$ isolates were of non albicans candida. The sensitivity pattern of Candida for various antifungal drugs was fluconazole $86.51 \%$, voriconzole 95.50\%, caspofungin 93.28\%, micafungin 93.28\%, amphotericin B $91.01 \%$ and flucytosine was 89.88\%. Resistance pattern was more in Candida tropicalis species. Candida krusei was found resistance to fluconazole and voriconazole.
\end{abstract}

Conclusion: Species level identification and their antifungal sensitivity should be performed to achieve their better clinical results.

Key word: Candida. Vitek, Antifungal sensitivity, Candida species

\section{Introduction}

Fungal infections are major cause of morbidity and mortality in the immunocompromised individuals and Candida are among the most common pathogens in these patients. The incidence of 6.9 per 1000 intensive care unit (ICU) patients and $7.5 \%$ of ICU patients received antifungal therapy was reported in the recent study. ${ }^{1,2}$ Growing population of immunocompromised patients and advances in medical and surgical managements has contributed an increase in candidaemia. Other associated risk factors causing fungal infections, include exposure to broad spectrum antimicrobial agents, mucosal colonisation by candida spp. Indwelling vascular catheters and premature infants. ${ }^{3,4}$ Candidaemia increases mortality rates in the range of $20-49 \% .^{5,6}$

The Candidaemia and invasive candidiasis are defined in general from diagnosis to prophylaxis, empiric and pre emptive strategies to treatment. So far, the scientific community has not been able to accurately predict invasive candidiasis and thus to define populations that benefit from prophylaxis or early treatment. Although it is well known that treatment is being initiated too late in the majority of patients, identification of the optimal time point to commence antifungal therapy remains challenging ${ }^{7,8}$

Candida species belong to the normal microbiota of an individual's mucosal oral cavity, gastrointestinal tract and vagina are responsible for various clinical manifestations from mucocutaneous overgrowth to blood stream infections. ${ }^{9}$ These yeast are commensal in the healthy human and may cause systemic infection in immunocompromised situations.

The genus Candida is composed of heterogeneous group of organisms and more than 17 different Candida species are known to be the aetiological agents of human infections, however, more than $90 \%$ of invasive infections are caused by $\mathrm{C}$. albicans, C. tropicalis, C. glabrata, C. parapsilosis and C. krusei. ${ }^{10}$ Systemic infections due to yeasts and resistance to antifungals is on the rise in Indian hospitals. ${ }^{11}$ Candida albicans accounts for $40-60 \%$ of yeast isolates in the developed countries. ${ }^{12}$ whereas Indian reports shows an increased predominance of C. non albicans isolates. ${ }^{11}$ Increasing resistance to azoles and amphotericin B has been reported from India as well as from other countries. ${ }^{11,12}$

The potential clinical importance of species level identification has been recognised as Candida species differ in the expression of putative virulence factors and antifungal susceptibility. ${ }^{13,14}$ Rapid identification also guides early appropriate antifungal therapy.

The aim of the present study is to identify the spectrum of Candida species in the clinical infections and to identify their sensitivity pattern to available antifungal agents. 


\section{Material And Method}

The study period was from 15 Jan. 2016 to 15 July 2016. All type of samples obtained from the ICU patients admitted in tertiary care centre Bareilly. Clinical specimens were endotracheal aspirates, sputum, urine and blood. Blood culture samples collected in blood culture bottles were incubated in Bactec automated blood culture system ( Becton Dickinson, USA) and up on getting a positive alarm, were sub cultured on to sabouraud dextrose agar (HiMedia, India) and blood agar plate after getting gram positive budding yeast cells on gram stain of blood culture broth. All other specimens were inoculated on to Sabouraud dextrose agar plates in addition to blood agar, chocolate agar and MacConkey agar (HiMedia, India). Suspected colonies of Candida were confirmed by Gram stain and then Identified with vitek 2 Compact (Biomerieux, France) using vitek 2 cards for identification of yeast and yeast like organisms (ID-YST cards) Kits. Antifungal susceptibility testing was performed with AST YS07 Kits on Vitek 2 Compact. Standard operative procedures as described by the manufacturer were followed. The study was done with prior approval from institutional ethical research committee.

\section{Result}

A total of 89 isolates of Candida species were obtained from different clinical specimens of I.C.U patients. Out of them, 48 isolates were from urine specimen, 26 from endotracheal tube aspirates, 7 from blood and 8 from sputum. The distribution and percentage of different candida species in the 89 isolates are given in the table. Accordingly, the species isolated were C. albicans $28(31.46 \%)$, C. tropicalis 41(46.06\%),C. guilliermondii 7(7.86\%), C. krusei 4(4.49\%), C. parasilopsis 3(3.3\%), C. glabrata 2(2.24\%), C. intermedia 2(2.24\%), C. lipolytica 1(1.12\%) and C. famata $1(1.12 \%)$.We observed that invasive candidiasis is more frequently caused by non albicans candida species $(68.53 \%)$ as compared to $\mathrm{C}$. albicans $(31.46 \%)$.

Table 1. The distribution and percentage of different candida species in different cinical isolates

\begin{tabular}{|c|c|c|c|c|c|}
\hline Isolates & Urine & E.T. & Sputum & Blood & Total \\
\hline Candida albicans & 16 & 8 & 2 & 2 & $28(31.46 \%)$ \\
\hline Candida tropicalis & 22 & 14 & 3 & 2 & $41(46.06 \%)$ \\
\hline $\begin{array}{l}\text { Candida } \\
\text { guilliermondii }\end{array}$ & 3 & 2 & 2 & 0 & $7(7.86 \%)$ \\
\hline Candida Krusei & 3 & 1 & 0 & 0 & $4(4.49 \%)$ \\
\hline $\begin{array}{c}\text { Candida } \\
\text { parapsilosis }\end{array}$ & 2 & 0 & 0 & 1 & $3(3.3 \%)$ \\
\hline Candida glabrata & 1 & 0 & 0 & 1 & $2(2.24 \%)$ \\
\hline Candida intermedia & 0 & 0 & 1 & 1 & $2(2.24 \%)$ \\
\hline Candida lipolytica & 0 & 1 & 0 & 0 & $1(1.12 \%)$ \\
\hline Candida famata & 1 & 0 & 0 & 0 & $1(1.12 \%)$ \\
\hline Total isolates & 48 & 26 & 8 & 7 & $89(100 \%)$ \\
\hline
\end{tabular}

The table 2 shows antifungal drug sensitivity pattern of Candida isolates in various samples.In this study antifungal drug sensitivity pattern of Candida isolates in various samples is following.

In the urine sample the candida albicans was $100 \%$ sensitive to fluconazole, voriconazole, amphotericin B and flucytosine except caspofungin and micafungin, both were $93.75 \%$ sensitive while non albicans candida is $75.0 \%$ sensitive to fluconazole, $96.8 \%$ to voriconazole, $87.5 \%$ to caspofungin. $87.5 \%$ to micafungin, $90.6 \%$ to amphotericin $\mathrm{B}$ and $81.25 \%$ sensitive to flucytosine.

E.T. aspirate candida albicans is $100 \%$ sensitive to all antifungal drugs and non albicans candida species are $75 \%$ sensitive to fluconazole, $75 \%$ sensitive to voriconazole, $100 \%$ sensitive to caspofungin, $100 \%$ sensitive to micafungin, $75 \%$ sensitive to amphotericin B and $87.5 \%$ sensitive to flucytosine.

In blood all the candida albicans and non albicans candida isolates were $100 \%$ sensitive to all the antifungal drugs

In sputum all candida albicans isolates were $100 \%$ sensitive to all antifungal drugs and non albicans candida isolates were $100 \%$ sensitive to all the antifungal drugs except amphotericin B which shows $83.3 \%$ sensitive.

Table 2.- Antifungal drug sensitivity pattern of Candida isolates in various samples

\begin{tabular}{|c|c|c|c|c|c|c|c|}
\hline Samples & & $\begin{array}{c}\text { Fluconazole } \\
\text { Sensitive }\end{array}$ & $\begin{array}{c}\text { Voriconazole } \\
\text { Sensitive }\end{array}$ & $\begin{array}{c}\text { Caspofungin } \\
\text { Sensitive }\end{array}$ & $\begin{array}{c}\text { Micafungin } \\
\text { Sensitive }\end{array}$ & $\begin{array}{c}\text { Amphotericin } \\
\text { B - Sensitive }\end{array}$ & $\begin{array}{c}\text { Flucytosine } \\
\text { Sensitive }\end{array}$ \\
\hline \multirow{3}{*}{ Urine 48} & C.albicans-16 & $100 \%$ & $100 \%$ & $93.75 \%$ & $93.75 \%$ & $100 \%$ & $100 \%$ \\
\cline { 2 - 8 } & C.nonalbican-32 & $75 \%$ & $96.8 \%$ & $87.5 \%$ & $87.5 \%$ & $90.6 \%$ & $81.25 \%$ \\
\hline \multirow{3}{*}{$\begin{array}{c}\text { E.T.Aspirate } \\
26\end{array}$} & C.albicans-8 & $100 \%$ & $100 \%$ & $100 \%$ & $100 \%$ & $100 \%$ & $100 \%$ \\
\cline { 2 - 8 } & C.nonalbicans-18 & $75 \%$ & $75 \%$ & $100 \%$ & $100 \%$ & $75 \%$ & $87.5 \%$ \\
\hline \multirow{3}{*}{$\begin{array}{c}\text { Blood 7 } \\
\text { Sputum 8 }\end{array}$} & C.albicans-2 & $100 \%$ & $100 \%$ & $100 \%$ & $100 \%$ & $100 \%$ & $100 \%$ \\
\cline { 2 - 9 } & C.nonalbicans-5 & $100 \%$ & $100 \%$ & $100 \%$ & $100 \%$ & $100 \%$ & $100 \%$ \\
\cline { 2 - 9 } & C.nonalbicans-6 & $100 \%$ & $100 \%$ & $100 \%$ & $100 \%$ & $100 \%$ & $100 \%$ \\
\hline
\end{tabular}

The table 3 Shows the resistance pattern of antifungal drugs among Candida species. Resistant for fluconazole, voriconazole, caspofungin, micafungin. amphotericin $\mathrm{B}$, flucytosine were $10.11 \%, 4.49 \%, 6.74 \%, 6.74 \%, 5.61 \%$ and $10.11 \%$ 
respectively. We observed that candida albicans was less resistant to all the drugs as compared to non albicans candida species.

Table 3. Resistance pattern of antifungal drugs among Candida species

\begin{tabular}{|c|c|c|c|}
\hline Drugs & $\begin{array}{c}\text { Candida } \\
\text { Albicans } 28\end{array}$ & $\begin{array}{c}\text { Non }- \text { albicans candida } \\
61\end{array}$ & $\begin{array}{c}\text { Total } \\
89\end{array}$ \\
\hline Fluconazole & $\begin{array}{c}\mathrm{S}-28(100 \%) \\
\mathrm{I}-0 \\
\mathrm{R}-0 \\
\text { Total-28 }\end{array}$ & $\begin{array}{c}\mathrm{S}-49(80.32 \%) \\
\mathrm{I}-3(4.91 \%) \\
\mathrm{R}-9(14.75 \%) \\
\text { Total }-61\end{array}$ & $\begin{array}{c}\mathrm{S}-77(86.51 \%) \\
\mathrm{I}-3(3.37 \%) \\
\mathrm{R}-9(10.11 \%) \\
\text { Total }-89\end{array}$ \\
\hline Voriconazole & $\begin{array}{c}\mathrm{S}-28(100 \%) \\
\mathrm{I}-0 \\
\mathrm{R}-0 \\
\text { Total-28 }\end{array}$ & $\begin{array}{c}\mathrm{S}-57(93.44 \%) \\
\mathrm{I}-0 \\
\mathrm{R}-4(6.55 \%) \\
\text { Total }-61\end{array}$ & $\begin{array}{c}\mathrm{S}-85(95.50 \%) \\
\mathrm{I}-0 \\
\mathrm{R}-4(4.49 \%) \\
\text { Total }-89\end{array}$ \\
\hline Caspofungin & $\begin{array}{c}\mathrm{S}-27(96.42 \%) \\
\mathrm{I}-0 \\
\mathrm{R}-(3.57 \%) \\
\text { Total- } 28\end{array}$ & $\begin{array}{c}\mathrm{S}-56(91.80 \%) \\
\mathrm{I}-0 \\
\mathrm{R}-5(8.1 \% \\
\text { Total }-61\end{array}$ & $\begin{array}{c}\mathrm{S}-83(93.25 \%) \\
\mathrm{I}-0 \\
\mathrm{R}-6(6.74 \%) \\
\text { Total }-89\end{array}$ \\
\hline Micafungin & $\begin{array}{c}\mathrm{S}-27(96.42 \%) \\
\mathrm{I}-0 \\
\mathrm{R}-1(3.57 \%) \\
\text { Total- } 28\end{array}$ & $\begin{array}{c}\mathrm{S}-56(91.80 \%) \%) \\
\mathrm{I}-0 \\
\mathrm{R}-5(8.19 \%) \\
\text { Total }-61\end{array}$ & $\begin{array}{c}\mathrm{S}-83(93.25 \%) \\
\mathrm{I}-0 \\
\mathrm{R}-6(6.74 \%) \\
\text { Total }-89\end{array}$ \\
\hline Amphotericin B & $\begin{array}{c}\mathrm{S}-28(100 \%) \\
\mathrm{I}-0 \\
\mathrm{R}-0 \\
\text { Total-28 }\end{array}$ & $\begin{array}{c}\mathrm{S}-53(86.88 \%) \\
\mathrm{I}-3(4.91 \%) \\
\mathrm{R}-5(8.19 \%) \\
\text { Total }-61\end{array}$ & $\begin{array}{c}\mathrm{S}-81(91.01 \%) \\
\mathrm{I}-3(3.37 \%) \\
\mathrm{R}-5(5.61 \%) \\
\text { Total }-89\end{array}$ \\
\hline Flucytosine & $\begin{array}{c}\mathrm{S}-28(100 \%) \\
\mathrm{I}-0 \\
\mathrm{R}-0 \\
\text { Total-28 }\end{array}$ & $\begin{array}{c}\mathrm{S}-52(85.24 \%) \\
\mathrm{I}-0 \\
\mathrm{R}-9(14.75 \%) \\
\text { Total }-61\end{array}$ & $\begin{array}{c}\mathrm{S}-80(89.88 \%) \\
\mathrm{I}-0 \\
\mathrm{R}-9(10.11 \%) \\
\text { Total }-89\end{array}$ \\
\hline
\end{tabular}

The table 4 shows antifungal susceptibility of different Candida species for individual antifungal drug

\begin{tabular}{|c|c|c|c|c|c|c|}
\hline $\begin{array}{l}\text { Species } \\
\text { C.albicans -28 }\end{array}$ & $\begin{array}{l}\text { Fluconazole } \\
\text { S-28(100\%) } \\
\text { I - } 0 \% \\
\text { R - } 0 \% \\
\text { Total -28 }\end{array}$ & $\begin{array}{l}\text { Voriconazole } \\
\text { S-28(100\%) } \\
\text { I - } 0 \% \\
\text { R - } 0 \% \\
\text { Total }-28\end{array}$ & $\begin{array}{l}\text { Caspofungin } \\
\text { S -27(96.42\%) } \\
\text { I - 0\% } \\
\text { R-1 }(3.57 \%) \\
\text { Total }-28\end{array}$ & $\begin{array}{l}\text { Micafungin } \\
\text { S-27(96.42\%) } \\
\text { I - } 0 \% \\
\text { R-1 }(3.57 \%) \\
\text { Total }-28\end{array}$ & $\begin{array}{l}\text { Amphotericin } \\
\text { S- } 28(100 \%) \\
\text { I - } 0 \% \\
\text { R - } 0 \% \\
\text { Total -28 }\end{array}$ & $\begin{array}{l}\text { Flucytocine } \\
\text { S-28(100\%) } \\
\text { I - 0\% } \\
\text { R - 0\% } \\
\text { Total -28 }\end{array}$ \\
\hline C.tropicalis - 41 & $\begin{array}{l}\text { S-36(87.80\%) } \\
\text { I-1 }(2.43 \%) \\
\text { R-4(9.75\%) } \\
\text { Total- } 41\end{array}$ & $\begin{array}{l}\text { S-38(92.68\%) } \\
\text { I-0\% } \\
\text { R-3 }(7.31 \%) \\
\text { Total- } 41\end{array}$ & $\begin{array}{l}\text { S- 36(87.80\%) } \\
\text { I- 0\% } \\
\text { R-5(12.19\%) } \\
\text { Total - 41 }\end{array}$ & $\begin{array}{l}\text { S- 36(87.80\%) } \\
\text { I -0\% } \\
\text { R -5(12.19\%) } \\
\text { Total - } 41\end{array}$ & $\begin{array}{l}\text { S-35(85.36\%) } \\
\mathrm{I}-2(4.87 \%) \\
\mathrm{R}-4(9.75 \%) \\
\text { Total }-41\end{array}$ & $\begin{array}{l}\mathrm{S}-36(85.36 \%) \\
\mathrm{I}-0 \% \\
\mathrm{R}-5(12.19 \%) \\
\text { Total }-41\end{array}$ \\
\hline $\begin{array}{l}\text { C.guilleirmondii - } \\
7\end{array}$ & $\begin{array}{l}\text { S }-4(57.14 \%) \\
\text { I }-2(28.57 \%) \\
\text { R- }-1(14.28 \%) \\
\text { Total- } 7\end{array}$ & $\begin{array}{l}\text { S- } 6(85.71 \%) \\
\text { I - 0\% } \\
\text { R- } 1(14.28 \%) \\
\text { Total }-7\end{array}$ & $\begin{array}{l}\mathrm{S}-7(100 \%) \\
\mathrm{I}-0 \% \\
\mathrm{R}-0 \% \\
\text { Total }-7\end{array}$ & $\begin{array}{l}\text { S-7(100\%) } \\
\mathrm{I}-0 \% \\
\mathrm{R}-0 \% \\
\text { Total }-7\end{array}$ & $\begin{array}{l}\mathrm{S}-7(100 \%) \\
\mathrm{I}-0 \% \\
\mathrm{R}-0 \% \\
\text { Total }-7\end{array}$ & $\begin{array}{l}\mathrm{S}-7(100 \%) \\
\mathrm{I}-0 \% \\
\mathrm{R}-0 \% \\
\text { Total }-7\end{array}$ \\
\hline C.krusei -4 & $\begin{array}{l}\mathrm{S}-0 \% \\
\mathrm{I}-0 \% \\
\mathrm{R}-4(100 \%) \\
\text { Total - } 4\end{array}$ & $\begin{array}{l}\text { S- } 4(100 \%) \\
\text { I- } 0 \% \\
\text { R- } 0 \% \\
\text { Total }-4 \\
\end{array}$ & $\begin{array}{l}\mathrm{S}-4(100 \%) \\
\mathrm{I}-0 \% \\
\mathrm{R}-0 \% \\
\text { Total- } 4\end{array}$ & $\begin{array}{l}\mathrm{S}-4(100 \%) \\
\mathrm{I}-0 \% \\
\mathrm{R}-0 \% \\
\text { Total }-4\end{array}$ & $\begin{array}{l}\mathrm{S}-4(100 \%) \\
\mathrm{I}-0 \% \\
\mathrm{R}-0 \% \\
\text { Total - } 4 \\
\end{array}$ & $\begin{array}{l}\mathrm{S}-0 \% \\
\mathrm{I}-0 \% \\
\mathrm{R}-4(100 \%) \\
\text { Total-4 } \\
\end{array}$ \\
\hline $\begin{array}{l}\text { C.parasilopsis - } \\
3\end{array}$ & $\begin{array}{l}\text { S- 3(100\%) } \\
\mathrm{I}-0 \% \\
\mathrm{R}-0 \% \\
\text { Total }-3 \\
\end{array}$ & $\begin{array}{l}\text { S- 3(100\%) } \\
\text { I-0\% } \\
\text { R-0\% } \\
\text { Total }-3 \\
\end{array}$ & $\begin{array}{l}\mathrm{S}-3(100 \%) \\
\mathrm{I}-0 \% \\
\mathrm{R}-0 \% \\
\text { Total }-3 \\
\end{array}$ & $\begin{array}{l}\text { S-3(100\%) } \\
\mathrm{I}-0 \% \\
\mathrm{R}-0 \% \\
\text { Total - } 3\end{array}$ & $\begin{array}{l}\text { S- 3(100\%) } \\
\text { I-0\% } \\
\text { R-0\% } \\
\text { Total - } 3\end{array}$ & $\begin{array}{l}\text { S -3(100\%) } \\
\mathrm{I}-0 \% \\
\mathrm{R}-0 \% \\
\text { Total }-3\end{array}$ \\
\hline C.glabrata -2 & $\begin{array}{l}\text { S- } 2(100 \%) \\
\text { I- }-0 \% \\
\text { R -0\% } \\
\text { Total -2 }\end{array}$ & $\begin{array}{l}\mathrm{S}-2(100 \%) \\
\mathrm{I}-0 \% \\
\mathrm{R}-0 \% \\
\text { Total }-2\end{array}$ & $\begin{array}{l}\mathrm{S}-2(100 \%) \\
\mathrm{I}-0 \% \\
\mathrm{R}-0 \% \\
\text { Total }-2\end{array}$ & $\begin{array}{l}\mathrm{S}-2(100 \%) \\
\mathrm{I}-0 \% \\
\mathrm{R}-0 \% \\
\text { Total }-2\end{array}$ & $\begin{array}{l}\text { S - 2(100\%) } \\
\mathrm{I}-0 \% \\
\mathrm{R}-0 \% \\
\text { Total }-2 \\
\end{array}$ & $\begin{array}{l}\mathrm{S}-2(100 \%) \\
\mathrm{I}-0 \% \\
\mathrm{R}-0 \% \\
\text { Total }-2\end{array}$ \\
\hline C.intermedia - 2 & $\begin{array}{l}\text { S-2(100\%) } \\
\text { I- }-0 \% \\
\text { R- } 0 \% \\
\text { Total }-2 \\
\end{array}$ & $\begin{array}{l}\text { S- } 2(100 \%) \\
\mathrm{I}-0 \% \\
\mathrm{R}-0 \% \\
\text { Total }-2 \\
\end{array}$ & $\begin{array}{l}\text { S- } 2(100 \%) \\
\text { I - } 0 \% \\
\text { R- } 0 \% \\
\text { Total - } 2 \\
\end{array}$ & $\begin{array}{l}\text { S- } 2(100 \%) \\
\text { I- }-0 \% \\
\text { R- } 0 \% \\
\text { Total }-2 \\
\end{array}$ & $\begin{array}{l}\mathrm{S}-2(100 \%) \\
\mathrm{I}-0 \% \\
\mathrm{R}-0 \% \\
\text { Total - } 2 \\
\end{array}$ & $\begin{array}{l}\mathrm{S}-2(100 \%) \\
\mathrm{I}-0 \% \\
\mathrm{R}-0 \% \\
\text { Total } 2\end{array}$ \\
\hline C.lipolytica - 1 & $\begin{array}{l}\text { S- } 1(100 \%) \\
\mathrm{I}-0 \% \\
\mathrm{R}-0 \% \\
\text { Total }-1\end{array}$ & $\begin{array}{l}\text { S- } 1(100 \%) \\
\mathrm{I}-0 \% \\
\mathrm{R}-0 \% \\
\text { Total }-1\end{array}$ & $\begin{array}{l}\mathrm{S}-1(100 \%) \\
\mathrm{I}-0 \% \\
\mathrm{R}-0 \% \\
\text { Total }-1\end{array}$ & $\begin{array}{l}\mathrm{S}-1(100 \%) \\
\mathrm{I}-0 \% \\
\mathrm{R}-0 \% \\
\text { Total }-1\end{array}$ & $\begin{array}{l}\mathrm{S}-!(100 \%) \\
\mathrm{I}-0 \% \\
\mathrm{R}-0 \% \\
\text { Total }-1\end{array}$ & $\begin{array}{l}\text { S -1(100\%) } \\
\mathrm{I}-0 \% \\
\mathrm{R}-0 \% \\
\text { Total }-1\end{array}$ \\
\hline
\end{tabular}




\section{Discussion}

Infections represents a frequent complication among patients admitted to tertiary care hospitals. In particular, the incidence of candidiasis has been increasing during the past few years. Infections with these yeasts also have a direct impact on the choice of empiric antifungal therapy and clinical outcome. Prior knowledge of species distribution in clinical isolates and drug sensitivity pattern among species help the clinician to choose early empirical therapy.

In the present study, we observed that non albicans Candida species had predominance over C. albicans, which is consistent with the published report from different parts of the world. ${ }^{15,16,17} \mathrm{C}$. tropicalis was the most common isolate in all samples, followed by C. albicans. A relatively greater proportion of C. tropicalis isolates in our study is concordant with other studies from India. ${ }^{18,19}$ Furthermore, invasive Candida infection was mostly caused by non albicans candida.

Candida species differ in their susceptibility to antifungal agents. All the strain of candida albicans were sensitive to all the antifungal drugs in all types of sample except one strain in urine sample was resistant for caspofungin and micafungin. Candida tropicalis were most resistant to all the antifungal drugs in urine sample. The resistant pattern of Candida tropicalis in urine sample for fluconazole $18.1 \%$, voriconazole $4.5 \%$, caspofungin $13.6 \%$, micafungin $13.6 \%$, Amphotericin B $18.1 \%$ and flucytosine $22.7 \%$.

Resistant pattern of Candida tropicalis in endotracheal aspirate was fluconazole $12.5 \%$, voriconazole $18.7 \%$, Amphotericin B $18.7 \%$ and flucytosine $12.5 \%$ while was $100 \%$ sensitive to caspofungin and micafungin. Candida krusei was resistant to fluconazole and voriconazole.

Some strains of Candida guilliermondii were shown resistance to fluconazole in urine, resistance to fluconazole and voriconazole in ET aspirate. Some strains of Candida guilliermondii in ET aspirate and sputum were shown resistance to Amphotericin B. Candida glabrata and Candida parapsilosis were sensitive to all the antifungal drugs.

Khotari et al ${ }^{20}$ from North India reported the susceptibility profile of Candida isolates as $92 \%$ were sensitive to AMB, $36 \%$ to fluconazole, $24 \%$ to itraconazole, $56 \%$ to voriconazole and $96 \%$ to flucytosine whereas in another study from South India showed $75 \%$ sensitivity to fluconazole, $100 \%$ to voriconazole, $92 \%$ to Amphotericin B and $90 \%$ to flucytosine.

In our study the susceptibility pattern is that fluconazole was sensitive to $86.5 \%$, voriconazole to $95.5 \%$, caspofungin to $93.25 \%$, to micafungin $93.25 \%$, Amphotericin B $91.0 \%$ and flucytosine was sensitive to $89.88 \%$ the results of our study simultates with previous studies.

\section{Conclusion}

Among the fungal pathogens, Candida species other than Candida albicans are a major cause of morbidity in hospitalised patients. Species level identification of Candida and their antifungal sensitivity testing should be performed to achieve better clinical results. Early antifungal therapy improves the outcome.

\section{References}

[1]. Kett DH, Azoulav E, Echeverria, Vincent JL. Extended Prevalence of Infectionin ICU Study (EPIC II) Group of Investigators. Candida bloodstream infections in intensive care unit study. Crit Care Med 2011 ;39:665-70.

[2]. AzoulavE, Dupont H, Tabah A, Lortholary O, Stah JP, Francais A, et al. Systemic antifungal therapy in critically ill patients without invasive fungal infection.Crit Care Med 2012;40:813-22.

[3]. Conica E, Azzani AM, Conti M. Epidemiology, incidence and risk factors for invasive candidiasis in high risk patients. Drugs 2009;69:5-14.

[4]. Pfaller MA, Pappas PG, Wingard JR. Invasive fungal pathogens: Current epidemiological trends. Clin Infect Dis 2006;43:S3-14.

[5]. Gudlaugsson O, Gillepie S, Lee K, Vande Berg J, Hu J, Messer S, et al. Attributable mortality of nosocomial candidemia, revisited. Clin Infect Dis 2003;37:1172-7.

[6]. Arendrup MC, Sulim S, Holm A, Nielsen L, Nielsen SD, Knudsen JD, et al. Diagnostic issues, clinical characterist,ics, and outcomes for patients with fungemia. J Clin Microbiology 2011;49:3300-8.

[7]. Garey KW, Rege M, Pai MP, MingoDE, Suda KJ, Turpin RS, et al. Time to initiation of fluconazole therapy impacts mortality in patients with candidemia: A multi- institutional study. Clin Infect Dis 2006;43:25-31.

[8]. Kumar A, Ellis P, Arabi Y, Roberts D, Light B, Parrilo JE, et al. Cooperative Antimicrobial Therapy of Septic Shock Database Research Group. Initiation of inappropriate antimicrobial therapy resuls in five fold reduction of survival in human septic shock. Chest 2009;136:1237-48.

[9]. Shao LC, Sheng CQ, Zhang WN. Recent advances in the study of antifungal lead compounds with new chemical scaffolds. Yao Xue Xue Bao 2007; 42:1129-36.

[10]. Pfaller MA, Diekema DJ, Procop GW, Rinaldi MG. Multicenter comparision of the VITEK 2 antifungal susceptibility test with the CLSI broth microdilution reference method for testing amphotericin B, flucytosine and voriconazole against Candida species. J Clin Microbiol 2007;45:3522-8.

[11]. Chakrabati A, Mohan B, Shrivastava SK, Marak RS, Ghosh A, Ray P. Change in distribution and antifungal susceptibility of Candida species isolated from candidaemia cases in a tertiary care centre during 1996 -2000. Indian J Med Res 2002;116:5-12.

[12]. Pfaller MA, Diekema DJ, Jones RN, Messer SA, Hollis RJ. Trends in antifungal susceptibility of Candida species isolated from pediatric and adult patients with blood stream infections: SENETRY Antimicrobial Surveillence Programme, 1997 TO 2000. J Clin Microbiology 2002;40:852-6.

[13]. Murray MP, Zinchuk R, Larone DH. CHROM agar Candida as the sole primary medium for trapid assimilation of trehelose test. J Clin Microbiol 2005;43:1210-2.

[14]. Balie GS, Douglas LJ, Iron limited biofilm of Candida albicans and their susceptibility to amphotericin B. Antimicrobial Agents Chemothera 1998;42:2146-9. 
[15]. Mean M, Marchetti O, Calandra T. Bench - to -bedside review:Candida infections in the intensive care unit. Crit Care 2008;12:204.

[16]. Mokaddas EM, AI-Sweith NA, Khan ZU. Species distribution and antifungal susceptibility of Candida bloodstream isolates in Kuwait: A 10 year study. J Med Microbiol 2007;56:255-9.

[17]. Jain N, Mathur P, Misra MC, Behera B, Xess I, Sharma SP. Rapid identification of yeast isolates from clinical specimensin critically ill trauma ICU patients. J Lab Phyicians 2012;4:30-4.

[18]. Khothavade RJ, Kura MM, Valand AG,Panthaki MH. Candida tropicalis; Its prevalence, pathogenicity and increasing resistance to fluconazole. J Med Microbiol 2010;59:873-80.

[19]. Mathews MS, Samuel PR, Suresh M. Emergence of Candida tropicalis as a major cause of fungaemia in India. Mycoses 2001;44:278-80.

[20]. Kothari A, Sagar V. Epidemiology of Candida bloodstream infections in a tertiary care institute in India. Indian J Med Microbiology 2008;27:171-2. 